\title{
ANÁLISE MICROBIOLÓGICA DE COPRODUTOS VITIVINÍCOLAS COM POTENCIAL PARA UTILIZAÇÃO NA ALIMENTAÇÃO DE RUMINANTES
}

\author{
MONTEIRO, Alegani Vieira ${ }^{1}$; \\ GONÇALVES, Fernanda Medeiros ${ }^{2}$; \\ PEREIRA, Rubens Alves ${ }^{1}$; \\ BRUSTOLIN, Joice Magali ${ }^{2}$; \\ AMARAL, Flavia Plucani ${ }^{1}$; \\ HALFEN, Jessica ${ }^{1}$; \\ CORRÊA, Márcio Nunes ${ }^{1}$.
}

Recebido: 16/09/2014

Aceito: 02/04/2015

\footnotetext{
${ }^{1}$ Núcleo de Pesquisa, Ensino e Extensão em Pecuária (NUPEEC), Departamento de Clínicas Veterinárias, Universidade Federal de Pelotas; ${ }^{2}$ Gestão Ambiental em Produção Animal (GAPA), Curso de Gestão Ambiental, Universidade Federal de Pelotas.
}

\section{RESUMO}

A agroindústria gera importante quantidade de resíduos e a área vinícola enfrenta problemas com o descarte. No processo da vitivinificação são gerados vários coprodutos, como engaço e bagaço, sendo os mais importantes e de maiores volumes. O setor vinícola do Rio Grande do Sul produziu aproximadamente 43 mil toneladas de bagaço em um período de três a quatro meses em 2013. Neste estudo, foram realizadas análises microbiológicas do bagaço de uvas in natura e após o processamento na forma de farinha, objetivando determinar o perfil microbiológico de coprodutos vitivinícolas. Foram isoladas bactérias do gênero Bacillus, sendo identificadas as espécies $B$. brevis, $B$. coagulans, $B$. mycoides e $B$. stearothermophilus. As características microbiológicas dos coprodutos vinícolas sugerem que estes alimentos possuem potencial de atuar como probióticos nas dietas para ruminantes. No entanto, são necessários estudos complementares para dimensionar os limites da sua utilização bem como as respostas na promoção da saúde animal.

Palavras-chave: Alimentação animal. Bacillus spp. Coprodutos da uva. Probióticos. 


\section{INTRODUÇÃO}

O Brasil é um dos países com maior atividade agrícola do mundo e, consequentemente, é um dos que mais produzem resíduos agroindustriais, fazendo com que os produtores e as indústrias enfrentem constantemente problemas com o descarte dos resíduos gerados, os quais tendem a se tornar uma fonte poluidora para o meio ambiente (CATANEO et al., 2008). Na produção de vinhos, a maior parte do bagaço de uva gerado é tratado como resíduo de baixo valor, entretanto este pode vir a ser utilizado para a alimentação animal (ROCKENBACH et al., 2011). Estima-se que 100 litros de vinho gerem cerca de 31,7 kg de resíduo, sendo cerca de $20 \mathrm{~kg}$ de bagaço de uva, sem considerar os demais resíduos. Desta forma, o alto volume de coprodutos gerados nas vinícolas resulta em sérios problemas de armazenagem, de transformação e/ou de eliminação, impactando ecológica e economicamente (CAMPOS, 2005).

A utilização destes coprodutos poderia representar um avanço significativo na manutenção do equilíbrio ambiental (ALONSO et al., 2002), pois os resíduos agroindustriais representam um recurso passível de aproveitamento na alimentação animal, capazes de contribuir para atender às exigências nutricionais, num contexto de viabilidade econômica e disponibilidade. Além disso, sua utilização na nutrição animal está totalmente de acordo com os princípios da conservação do meio ambiente (TEIXEIRA et al., 2007), pois são materiais secundários gerados no processo de industrialização de produtos agrícolas e que tem potencial mercadológico (BURGI, 1986).

O estado do Rio Grande do Sul é o maior produtor de vinho do Brasil, com uma produção de mais de 600 milhões de quilos de uva em 2014 (IBRAVIN, 2014). Observou-se que, num período de três a quatro meses, o montante de bagaço gerado representou quase $20 \%$ das uvas colhidas (ROCKENBACH et al., 2011). Os coprodutos do processo de vitivinificação gerados em maior quantidade são: o bagaço, constituído pelas cascas e sementes residuais ao processo de esmagamento, e o engaço, material representado pelo talos dos cachos (SILVA, 2003). Atualmente, suas utilizações se limitam a adubo para os vinhedos, destino pouco nobre e que vem despertando descontentamento dos orgãos ambientais pois, na maioria dos casos, não recebem tratamento prévio ao descarte (FLUCK, 2013). 
Barroso et al. (2006) citam que estes coprodutos podem representar uma boa opção como complemento alimentar de ruminantes, por suas características nutricionais, como a alta concentração de carboidratos fibrosos e uma adequada fração de proteína bruta. Muitos estudos vem sendo realizados sobre a utilização destes coprodutos para a alimentação animal (FURTADO et al., 2011; GESSNER et al., 2013; KLINGER et al., 2013; LAVEZZO et al., 1990; LOUSADA et al., 2005), tanto para ruminantes como para monogástricos. Ainda, alguns autores já verificaram a atividade antibacteriana de compostos fenólicos da uva in vitro (ARVANITOYANNIS et al., 2006; BAYDAR et al., 2004; JAYAPRAKASHA et al., 2003; RHODES et al., 2006; ROTAVA et al., 2009), constatando também que estes coprodutos se constituem em fontes naturais antioxidantes em comparação aos sintéticos, largamente utilizados, e sem efeitos indesejáveis em processos enzimáticos ou orgânicos.

Um fator que vem limitando o uso desses coprodutos é o alto teor de umidade contido, o que consequentemente favorece a proliferação de microrganismos (WEIBERG, 1992), levando a uma deterioração em um curto período de tempo. Para isso, Ashbell et al. (1997) indicam como solução o armazenamento na forma de silagem, e outros trabalhos também vêm sendo realizados para elucidar estas questões ainda não sanadas, ainda que a perspectiva, corroborada por alguns resultados, seja favorável. Durante o processo de silagem, diversos tipos de bactérias podem vir a se desenvolver nestes produtos, dentre as quais incluem-se as com potencial benéfico para animais, como alguns tipos de Bacillus spp. As frutas e seus derivados são em geral alimentos ácidos e a elevada acidez restringe a microbiota deterioradora, especialmente os microrganismos patogênicos (SIQUEIRA; BORGES, 1997). Bactérias e fungos fazem parte da microbiota dominante de frutas e vegetais em geral (BEUCHAT, 1996), sendo que a maior parte dos contaminantes das frutas reside em sua parte externa.

O gênero Bacillus é compreendido por microrganismos Gram-positivos, com forma de bastonetes, formadores de esporos, aeróbios ou anaeróbios facultativos, que podem ser encontrados no solo, na água, na poeira e no ar (TAM et al., 2006). Poucas espécies são consideradas patogênicas, outras apresentam significância ambiental, industrial e clínica (FRANCO; LANDGRAF, 2002). De acordo com TAM et al. (2006), os bacilos vêm sendo 
utilizados como probióticos, termo este que refere-se a uma preparação ou a um produto que contenha cepas de microrganismos viáveis em quantidades suficientes para produzir efeitos benéficos para o hospedeiro (FUSTER; GONZÁLEZ-MOLERO, 2007), tais como um equilíbrio da microbiota intestinal (SANDERS, 2003). Esse equilíbrio promove aumento da população de espécies bacterianas capazes de sintetizar proteínas e vitaminas (ORTIZ-RUBIO et al., 2009). Diversas pesquisas têm destacado a importância dos aditivos alimentares, tais como os probióticos, que interferem no metabolismo melhorando a eficiência de utilização dos alimentos e proporcionando um incremento na produção animal (BEZERRA et al., 2010; FRANÇA; RIGO, 2011; TIMMERMAN et al., 2005; ZEOULA et al., 2008).

Assim sendo, o objetivo deste trabalho foi analisar o perfil microbiológico de coprodutos vitivinícolas in natura e após processamento (farinha).

\section{MATERIAL E MÉTODOS}

\section{Obtenção das amostras}

O estudo foi realizado em abril de 2014, utilizando-se coprodutos de uva gerados no processo de fabricação do vinho, cedidos por uma empresa vinícola do sul do Rio Grande do Sul. Os coprodutos foram coletados aleatoriamente na vinícola sendo depois armazenados em sacos plásticos e identificados. Coletou-se o bagaço, constituído pelas cascas e sementes residuais ao processo de esmagamento e o engaço, material representado pelos talos dos cachos das uvas. As amostras permaneceram armazenadas sob refrigeração a $-18^{\circ} \mathrm{C}$.

\section{Processamento das amostras}

As amostras in natura não sofreram tratamento térmico. As amostras que serviram de substrato para a produção da farinha foram processadas realizando-se a secagem em estufa com ar forçado (Marconi, Modelo MA 680) a $56{ }^{\circ} \mathrm{C}$ por 24 horas, sendo posteriormente realizada a moagem em moinho de facas (Tipo Willey, Tecnal, Modelo TE 680). A separação granulométrica foi realizada com o auxilio de peneira de $1 \mathrm{~mm}$. 


\section{Análises microbiológicas}

As análises microbiológicas foram realizadas no laboratório de Doenças Infecciosas da Faculdade de Veterinária (UFPEL), utilizando-se os meios de cultura Ágar Brain Heart Infusion (BHI-DIFCO), Ágar MacConkey (DIFCO), Ágar Chapman (DIFCO) e Ágar Sabouraud (DIFCO), os quais são considerados meios de utilização geral, adequados para a cultura de uma ampla variedade de microrganismos. As amostras foram separadas em oito grupos: GEgrupo engaço; GBUB-grupo bagaço de uva branca; GBUR-grupo bagaço de uva roxa; GBURF-grupo bagaço de uva roxa fermentada; GFE-grupo farinha do engaço; GFBUB-grupo farinha do bagaço de uva branca; GFBUR-grupo farinha do bagaço de uva roxa e GFBURFgrupo farinha do bagaço de uva roxa fermentada (Figura 1).

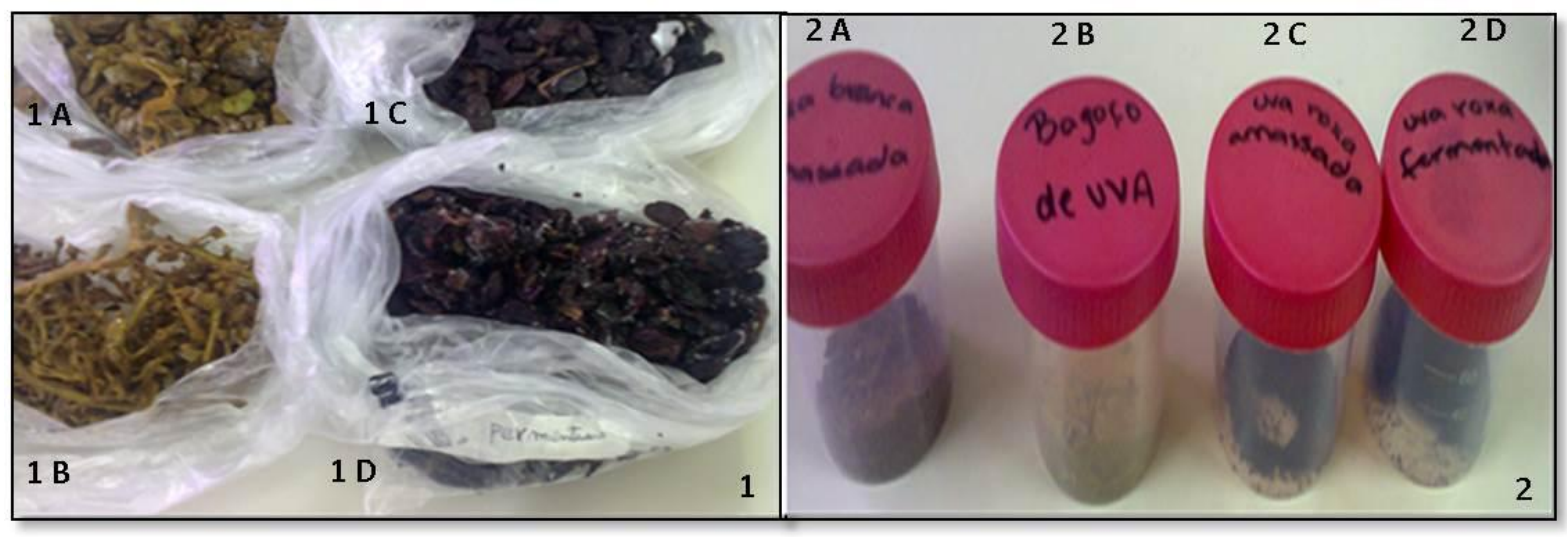

Figura 1 - Amostras de uva in natura (1) e em farinha após processamento (2). 1A: GBUB-grupo bagaço de uva branca; 1B: GE-grupo engaço; 1C: GBUR-grupo bagaço de uva roxa e 1D: GBURF-grupo bagaço de uva roxa

fermentada. 2A: GFBUB-grupo farinha do bagaço de uva branca; 2B: GFE-grupo farinha do engaço; 2C: GFBUR-grupo farinha do bagaço de uva roxa e 2D: GFBURF-grupo farinha do bagaço de uva roxa fermentada.

Conforme Zenebon et al. (2008), pesou-se $1 \mathrm{~g}$ de material de cada grupo, adicionando-se 9 $\mathrm{mL}$ de caldo BHI. Após a semeadura, o material foi incubado em estufa bacteriológica a $37^{\circ} \mathrm{C}$ por 24 h. Após esse período, realizou-se a técnica de coloração de Gram. As amostras foram analisadas em triplicata. A caracterização bioquímica das amostras foi realizada usando as reações de fermentação dos seguintes substratos: glicose, galactose, manose, rafinose, xilose, urease, celobiose, salicina, sorbitol e manitol. Também foi avaliada a produção da 
oxidase, catalase, indol, nitrato, $\mathrm{H}_{2} \mathrm{~S}$, e utilização de citrato. Todas as provas bioquímicas foram realizadas como descrito por Barrow e Feltham (1993).

\section{RESULTADOS E DISCUSSÃO}

A literatura nacional para ruminantes relata o uso eficiente de inúmeros coprodutos e alimentos alternativos na forma de silagem, grão, feno, casca e resíduo seco ou in natura (café, uva, abacaxi, maracujá, entre outros) que poderiam, após pesquisas, ser incluídos nas dietas dos animais (FURTADO et al., 2011).

Nos coprodutos identificados como GE, GFBUB, e GFBURF não houve crescimento microbiano. Dos outros coprodutos: GBUB, GBUR, GBURF, GFE, GFBUR foram isolados microrganismos do gênero Bacillus spp. Os microrganismos isolados dos coprodutos vitivinícolas estão apresentados na Tabela 1.

Tabela 1 - Microrganismos isolados nos coprodutos vitivinícolas procedentes de uma vinícola do sul do Rio Grande do Sul, 2014.

\begin{tabular}{l|l}
\hline \multicolumn{1}{c|}{ GRUPOS } & \multicolumn{1}{c}{ MICRORGANISMO } \\
\hline GE-engaço & Não houve crescimento \\
GBUB-bagaço de uva branca & $\begin{array}{l}\text { B. coagulans } \\
\text { B. mycoides } \\
\text { GBUR-bagaço de uva roxa }\end{array}$ \\
GBURF-bagaço de uva roxa fermentada & B. brevis \\
\hline GFE-farinha do engaço & B. stearothermophilus \\
GFBUB-farinha do bagaço de uva branca & Não houve crescimento \\
GFBUR-farinha do bagaço de roxa & B. mycoides \\
GFBURF-farinha do bagaço de uva roxa fermentada & Não houve crescimento \\
\hline
\end{tabular}

Segundo Chaud et. al. (2007), a faixa de temperatura ideal para o crescimento de bactérias do gênero Bacillus spp. é de 40 a $70{ }^{\circ} \mathrm{C}$ por um período de 14 a $96 \mathrm{~h}$, indicando que o tratamento térmico nas amostras processadas (farinha) provavelmente não alterou os resultados encontrados. Do mesmo modo, Gomes (2013) verificou que este gênero de 
bactérias possui a habilidade de formar esporos, o que aumenta a sua resistência térmica, sendo necessária uma maior temperatura para inativá-los. Santos et al. (2008) relatam que as frutas permanecem com o seu interior praticamente estéril, desde que não ocorra ruptura em alguma parte da casca, como o que ocorre durante os processamentos das uvas para produção de vinho, tornando então, os coprodutos gerados passíveis de contaminantes naturais presentes nas cascas.

Bactérias do gênero Bacillus têm sido cada vez mais utilizadas em produtos alimentares por apresentarem características probióticas comuns, tal como a viabilidade intestinal (TAM et al., 2006). Considerando que os bacilos B. coagulans e B. brevis, encontrados neste estudo, estão sendo usados como probióticos para consumo humano e animal (SOROKULOVA et al., 2008), os coprodutos do processo de vitivinificação poderiam atuar como promotores de saúde intestinal nos rebanhos, expandindo sua utilização além da funcionalidade nutricional.

No que se refere ao B. stearothermophilus, seus esporos são considerados convenientes indicadores biológicos da esterilização pelo calor úmido, particularmente à temperatura de referência de $121{ }^{\circ} \mathrm{C}$ (PENNA; MACHOSHVILI, 2003). Contudo, sua ação probiótica não foi evidenciada até o momento.

Os dados relacionados à avaliação microbiológica de resíduos agroindustriais ainda são escassos, sendo precoce a recomendação destes para a inserção nas dietas para ruminantes. Cândido et al. (2008), ressaltam que ainda são necessários novos estudos para a utilização de coprodutos na alimentação animal, principalmente em relação à métodos de tratamento, conservação e armazenagem, buscando assim aumentar e melhorar a viabilidade dos componentes.

O presente estudo verificou uma interessante oportunidade em utilizar os coprodutos da vitivinificação em dietas para ruminantes, aproveitando desta maneira o benefício decorrente da presença de bactérias com potencial probiótico. Estas evidências acumuladas sobre os benefícios decorrentes do uso dos probióticos justificam o aprofundamento dos estudos com coprodutos a fim de aperfeiçoar suas utilizações.

A utilização de coprodutos agroindustriais nas dietas para ruminantes torna-se uma característica economicamente e ambientalmente importante para diversas regiões 
produtoras de uvas à medida que diminui o impacto ambiental gerado pelo descarte inadequado destes resíduos.

\section{CONCLUSÃO}

As características microbiológicas dos coprodutos vinícolas sugerem que estes alimentos possuem potencial de atuar como probióticos nas dietas para ruminantes. No entanto, são necessários estudos complementares para dimensionar os limites da sua utilização bem como as respostas na promoção da saúde animal.

\section{MICROBIOLOGICAL ANALYSIS OF WINE CO-PRODUCTS WITH POTENTIAL FOR USE IN RUMINANT FEEDING}

\section{ABSTRACT}

T he agro-industry generates significant amount of waste and the winery sector faces problems with the disposal of that. In the winery process, multiple co-products are generated, such as pomace and stalks, being these the most important and with larger volumes. The wine industry of Rio Grande do Sul has produced about 43,000 tons of pomace in a period of three to four months in 2013. In this study microbiological analysis of pomace in natura and co-products obtained from the grapes were performed. Bacteria from the genus Bacillus were isolated, and the species B. brevis, $B$. coagulans, $B$. mycoides and $B$. stearothermophilus were identified, which have the potential to act as probiotics in animal feeding. It was concluded that the Bacillus species found in the stems and grape pomace are not considered pathogenic and therefore these co-products can be used in animal feeding. Therefore, complementary studies are necessary to measure the limits of their application, as well as their responses for the promotion of animal health.

Keywords: Animal Nutrition. Bacillus spp. Grape co-products. Probiotics. 


\section{ANÁLISIS MICROBIOLÓGICO DE SUBPRODUCTOS VITIVINÍCOLAS CON POTENCIAL PARA USO EN LA ALIMENTACIÓN DE RUMIANTES}

\section{RESUMEN}

a agroindustria genera gran cantidad de residuos y el área vinícola enfrenta problemas con el descarte. En el proceso de vitivinificación se generan varios subproductos, tales como tallos y orujo, siendo los volúmenes más importantes y más grandes. La industria del vino de Río Grande do Sul produjo aproximadamente 43.000 toneladas de bagazo en un período de tres a cuatro meses en 2013. En este estudio, se llevaron a cabo análisis microbiológico de orujo de uva in natura y después del procesamiento en forma de harina, con el objetivo determinar el perfil microbiológico de subproductos del vino. Las bacterias que se aislaron fueron del género Bacillus, siendo identificadas las especies $B$. brevis, $B$. coagulans, $B$. mycoides y $B$. stearothermophilus. Las características microbiológicas de los subproductos sugieren que estos alimentos poseen potencial de actuar como probióticos en las dietas de los rumiantes. A pesar de esto, son necesarios estudios complementarios para dimensionar los límites de su utilización, así como las respuestas en la promoción de la salud animal.

Palabras clave: Nutrición animal. Bacillus spp. Subproductos de la uva. Probióticos.

\section{AGRADECIMENTOS}

Ao Professor Doutor Fábio Pereira Leivas Leite do Laboratório de Bacteriologia do Centro de Biotecnologia da Universidade Federal de Pelotas-UFPEL, e à Médica Veterinária MSc Renata Costa Schramm do Laboratório de Doenças Infecciosas da Faculdade de Veterinária-UFPEL.

\section{REFERÊNCIAS}

ALONSO, A. M.; GUILLÉN, D. A.; BARROSO, C. G.; PUERTAS, B.; GARCÍA, A. Determination of antioxidant activity of wine by-products and it correlation with polyphenolic content. Journal of Agricultural and Food Chemistry, v. 50, p. 5832-5836, 2002.

ARVANITOYANNIS, I. S.; LADAS, D.; MAVROMATIS' A. Potential uses and applications of treated wine waste. International Journal of Food Science and Technology, v. 41, p. 475487, 2006. 
ASHBELL, G.; WEINBERG, Z. G.; BRUCKENTAL, I.; TABORI, K.; SHARET, N. Wheat Silage: Effect of Cultivar and Stage of Maturity on Yield and Degradability in Situ. Journal of Agricultural and Food Chemistry, v. 45, n. 3, p. 709-712, 1997.

BARROSO, D. D.; ARAÚJO, G. G. L.; SILVA, D. S.; MEDINA, F. T. Resíduo desidratado de vitivinícolas associado a diferentes fontes energéticas na alimentação de ovinos: consumo e digestibilidade aparente. Ciência e Agrotecnologia, v. 30, n. 4, p. 767-773, 2006.

BAYDAR, N. G.; OZKAN, G.; SAGDIÇ, O. Total phenolic contents and antibacterial activities of grape (Vitis vinifera L.) extracts. Food Control, v. 15, n. 5, p. 335-339, 2004.

BARROW, G. I.; FELTHAM, R. K. A. Manual for the identification of medical bacteria. 3. ed. Cambridge University Press, 1993, 331p.

BEUCHAT, L. R. Pathogenic microorganisms associated to fresh product. Journal of Food Protection, v. 59, p. 204-216, 1996.

BEZERRA, L. R.; SILVA, A. M. A.; AZEVEDO, S. A.; MENDES, R. S.; MANGUEIRA, J. M.; GOMES, A. K. A. Desempenho de cordeiros Santa Inês submetidos a aleitamento artificial enriquecido com Spirulina platensis. Ciência Animal Brasileira, Goiânia, v. 11, n. 2, p. 258-263, 2010.

BURGI, R. Utilização de subprodutos agroindustriais na alimentação de ruminantes. In: CONGRESSO BRASILEIRO DE PASTAGENS, 8, 1986, Piracicaba. Anais. Piracicaba: FEALQ, 1986, p. 101-117.

CÂNDIDO, M. J. D.; BOMFIM, M. A. D.; SEVERINO, L. S.; OLIVEIRA, S. Z. R. Utilização de coprodutos da mamona na alimentação animal. In: CONGRESSO BRASILEIRO DE MAMONA: ENERGIA E RICINOQUÍMICA, 3, 2008, Salvador. Anais. Salvador, 2008.

CAMPOS, L. M. A. S. Obtenção de extratos de bagaço de uva Cabernet Sauvignon (Vitis vinifera): parâmetros de processo e modelagem matemática. Florianópolis: UFSC, 2005. 123p. Dissertação (Mestrado em Engenharia de Alimentos), Departamento de Engenharia Química e Engenharia de Alimentos, Universidade Federal de Santa Catarina, 2005.

CATANEO, C. B.; CALIARI, V.; GONZAGA, L. V.; KUSKOSKI, E. M.; FETT, R. Atividade antioxidante e conteúdo fenólico do resíduo agroindustrial da produção de vinho. Semina: Ciências Agrárias, Londrina, v. 29, n. 1, p. 93-102, 2008.

CHAUD, L. C. S.; VAZ, P. V.; FELIPE, M. G. Considerações sobre a produção microbiana e aplicações de proteases. Revista Nucleus, v. 4, n. 1-2, p. 87-97, 2007.

FLUCK, A. C.; COSTA, O. I. D.; FIOREZE, V. I.; ROSA. P. P.; RIZZO, F. A.; ALFAYA JUNIOR, H. Utilização de subprodutos da indústria vinícola na dieta de ruminantes: bagaço de uva. In: SIMPÓSIO DE SUSTENTABILIDADE E CIÊNCIA ANIMAL, 3, 2013, São Paulo. Anais. São Paulo, 2013. 
FRANÇA, R. A.; RIGO; E. J. Utilização de leveduras vivas (Saccharomyces cerevisiae) na nutrição de ruminantes. FAZU em Revista, Uberaba, n. 8, p. 187-195, 2011.

FRANCO, B. D. G. M.; LANDGRAF, M. Microbiologia dos alimentos. São Paulo: Atheneu, 2002. 182p.

FURTADO, C. E.; BRANDI, R. A.; RIBEIRO, L. B. Utilização de coprodutos e demais alimentos alternativos para dietas de equinos no Brasil. Revista Brasileira de Zootecnia, v. 40, p. 232241, 2011.

FUSTER, G. O.; GONZÁLEZ-MOLERO, I. Probióticos y prebióticos en la prática clínica. Nutrición Hospitalaria, Málaga, v. 22, n. 2, p. 26-34, 2007.

GESSNER, D. K.; FIESEL, A.; MOST, E.; DINGES, J.; WEN, G.; RENGSEIS, R.; EDER, K. Supplementation of a grape seed and grape marc meal extract decreases activities of the oxidative stress-responsive transcription factors NF-kB and Nrf2 in the duodenal mucosa of pigs. Acta Veterinaria Scandinavica, v. 55, n. 18, 2013.

GOMES, M. J. P. Tópicos em Bacteriologia Veterinária. FAVET-UFRGS, 2013. Disponível em: <http://www.ufrgs.br/labacvet/files/G\%C3\%AAnero\%20Bacillus\%204-20131\%20vers\%C3\%A3o\%202013.pdf> Acesso em: 22 abril 2014.

IBRAVIN (INSTITUTO BRASILEIRO DO VINHO). Cadastro Vinícola. Disponível em: <http://www.ibravin.org.br/noticias/161-safra-2014-fecha-em-mais-de-600-milhoes-dequilos-de-uva>. Acesso em: julho de 2014.

JAYAPRAKASHA, G. K.; SELVI. T.; SAKARIAH, K. K. Antibacterial and antioxidant activities of grape (Vitis vinifera) seed extracts. Food Research International, v. 36, p. 117-122, 2003.

KLINGER, A. C. K.; TOLEDO, G. S. P.; SILVA, L. P.; MASCHKE, F.; CHIMAINSKI, M.; SIQUEIRA, L. Bagaço de uva como ingrediente alternativo no arraçoamento de coelhos em crescimento. Ciência Rural, v. 43, n. 9, p. 1654-1659, 2013.

LAVEZZO, O. E. N. M. Utilização de resíduos culturais e de beneficiamento na alimentação de bovinos: abacaxi, banana, caju, uva e maça. In: SIMPÓSIO SOBRE NUTRIÇÃO DE BOVINOS, 6. , 1990, Piracicaba. Anais. Piracicaba: Fundação de Estudos Agrários Luiz de Queiroz, sd. p. 746.

LOUSADA J. J. N. E.; RODRIGUEZ, N. M.; PIMENTEL, J. C. M.; LÔBO, R. N. B. Consumo e Digestibilidade de Subprodutos do Processamento de Frutas em Ovinos. Revista Brasileira de Zootecnia, v. 34, n. 2, p. 659-669, 2005. 
ORTIZ-RUBIO, M. A.; GALINA M. A.; PINEDA, L. J. Effect of slow nitrogen intake supplementation with or without a lactic probiotic on Pelibuey lamb growth. Nutritional and foraging ecology of sheep and goats, v. 85, p. 309-314, 2009.

PENNA, T. C. V.; MACHOSHVILI, I. A. Esterilização térmica. Conceitos Básicos da Cinética de Morte Microbiana. Revista Farmácia Bioquímica, Univ. São Paulo, v. 1, p. 1-5, 1997.

RHODES, P. L.; MITCHELL, J. W.; WILSON, M. W.; MELTON, L. D. Antilisterial activity of grape juice and grape extracts derived from Vitis vinifera variety Ribier. International Journal of Food Microbiology, v. 107, n. 3, p. 281-286, 2006.

ROCKENBACH, I. I.; RODRIGUES, E.; GONZAGA, L. V.; CALIARI, V.; GENOVESE, M. I.; GONÇALVES, A. E. S. S.; FETT, R. Phenolic compounds content and antioxidant activity in pomace from selected red grapes (Vitis vinifera L. and Vitis labrusca L.) widely produced in Brazil. Food Chemistry, v. 127, p. 174-179, 2011.

ROTAVA, R.; ZANELLA, I.; SILVA, L. P.; MANFRON, M. P.; CERON, C. S.; ALVES, S. H.; KARKOW, A. K.; SANTOS, J. P. A. Atividade antibacteriana, antioxidante e tanante de subprodutos de uva. Ciência Rural, v. 39, n. 3, p. 941-944, 2009.

SANDERS, M. E. Probiotics: considerations for human health. Nutrition Review, v. 6, n. 3, p. 91-99, 2003.

SANTOS, C. A. A.; COELHO, A. F. S.; CARREIRO, S. C. Avaliação microbiológica de polpas de frutas congeladas. Ciência e Tecnologia de Alimentos, v. 28, n. 4, p. 913-915, 2008.

SILVA, L. M. L. R. Caracterização dos Subprodutos da Vinificação. Revista do ISPV, n. 28, 2003.

SIQUEIRA, R. S.; BORGES, M. F. Microbiologia de frutas e produtos derivados. In: TORREZAN, R. (Coord.). Curso de processamento de frutas. Rio de Janeiro: EMBRAPA/CTAA, 1997. p. 213.

SOROKULOVA, I. B.; PINCHUK, I. V.; DENAYROLLES, M.; OSIPOVA, I. G.; HUANG, J. M.; CUTTING, S. M.; URDACI, M. C. The Safety of Two Bacillus Probiotic Strains for Human Use. Digestive Diseases and Sciences, v. 53, p. 954-963, 2008.

TAM, N. K. M.; UYEN, N. Q.; HONG, H. A.; DUC, L. H.; HOA, T. T.; SERRA, C. R.; HENRIQUES, A. O.; CUTTING, S. M. The Intestinal Life Cycle of Bacillus subtilis and Close Relatives. Journal of . Bacteriology, v. 188, n. 7, p. 2692-2700, 2006.

TEIXEIRA, F. A.; PIRES, A. V.; NASCIMENTO, P. V. N. Bagaço de cana-de-açúcar na alimentação de bovinos. Veterinaria Organización España REDVET. Revista Electrónica de Veterinaria, v. 8, n. 6, p. 1-9, 2007. 
TIMMERMAN, H. M.; MULDER, L.; EVERTS, H.; VAN ESPEN, D. C.; VAN DER WAL, E.; KLAASSEN, G.; ROUWERS, S. M. G.; HARTEMINK, R.; ROMBOUTS, F. M.; BEYNEN, A. C. Health and growth of veal calves fed milk replacers with or without probiotics. Journal Dairy Science, v. 88, p. 2154-2165, 2005.

WEINBERG, Z. G. Bioconservation of agricultural by-products by ensiling. In: Simpósio sobre utilização de subprodutos agroindustriais e resíduos de colheita na alimentação de ruminantes. 1992, São Carlos. Anais. São Carlos. Embrapa, p. 191-198, 1992.

ZENEBON, O.; PASCUET, N. S.; TIGLEA, P. Métodos Físico-químicos para análise de alimentos. São Paulo: Instituto Adolfo Lutz, 2008. 1020p.

ZEOULA, L. M.; BELEZE, J. R. F.; GERON, L. J. V.; MAEDA, E. M.; PRADO, I. N.; PAULA, M. C. Digestibilidade parcial e total de rações com a inclusão de ionóforo ou probiótico para bubalinos e bovinos. Revista Brasileira de Zootecnia, v. 37, n. 3, p. 563-571, 2008. 